\title{
Phase densities and lamellar morphologies of semicrystalline polyethylenes via absolute small-angle X-ray scattering measurements
}

\section{Monika Basiura-Cembala, Kurt Erlacher, Jan Skov Pedersen and Bart Goderis}

J. Appl. Cryst. (2015). 48, 1498-1506

\section{\# IUCr Journals CRYSTALLOGRAPHY JOURNALS ONLINE \\ Copyright (C) International Union of Crystallography \\ Author(s) of this paper may load this reprint on their own web site or institutional repository provided that this cover page is retained. Republication of this article or its storage in electronic databases other than as specified above is not permitted without prior permission in writing from the IUCr. \\ For further information see http://journals.iucr.org/services/authorrights.html}




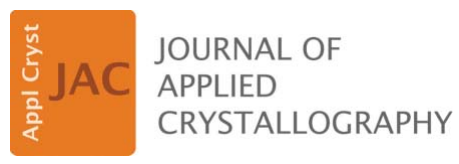

ISSN 1600-5767

Received 15 July 2014

Accepted 6 August 2015

Edited by V. T. Forsyth, Institut Laue-Langevin, France, and Keele University, UK

₹ Current address: Bruker AXS GmbH, Östliche Rheinbrückenstrasse 49, 76187 Karlsruhe, Germany

Keywords: small-angle X-ray scattering; absolute invariant; polyethylene; amorphous phases.

\section{Phase densities and lamellar morphologies of semicrystalline polyethylenes via absolute small- angle X-ray scattering measurements}

\author{
Monika Basiura-Cembala, ${ }^{a *}$ Kurt Erlacher, $^{\mathrm{b}} \neq$ Jan Skov Pedersen ${ }^{\mathrm{b}}$ and Bart Goderis ${ }^{\mathrm{c}}$ \\ anstitute of Textile Engineering and Polymer Materials, University of Bielsko-Biala, Willowa 2, Bielsko-Biala 43-309 \\ Poland, 'bepartment of Chemistry, University of Aarhus, Langelandsgade 140, Aarhus DK-8000 C, Denmark, and \\ ${ }^{\mathrm{c}}$ Chemistry Department, Catholic University of Leuven, Celestijnenlaan 200F, Heverlee B-3001, Belgium. *Correspon- \\ dence e-mail: mbasiura@ath.bielsko.pl
}

With the crystalline volume fraction from small-angle X-ray scattering (SAXS) and the density of the crystalline phase from wide-angle X-ray diffraction, the amorphous phase density of two representative polyethylenes was calculated as a function of temperature using the absolute total SAXS scattering power or invariant. The density of the amorphous phase in semicrystalline polyethylene is crystallinity independent and is lower than melt-extrapolated values reported in the literature. Model-independent SAXS-based crystallinity values can be calculated with the aid of the densities of the crystalline and amorphous phase and the absolute SAXS invariant. Such model-independent crystallinity values can be used in SAXS curve-shape analysis procedures to obtain the average thickness of the crystalline and amorphous layers also in the case of non-ideal lamellar semicrystalline polymer morphologies for which the number-average long period cannot be retrieved from the maxima in correlation functions or interface distribution functions.

\section{Introduction}

Crystallizable polymers tend to solidify into stacks of alternating nanometre-sized amorphous and crystalline layers that further aggregate into spherulitic entities, called spherulites (Bower, 2002). Polymer chain folds and larger loops remain amorphous and reside in between the crystalline layers together with microstructural and conformational defects, such as chain ends, noncrystallizable units and entanglements. Therefore, crystallizable polymers are semicrystalline with a given degree of crystallinity depending on the chain microstructure and crystallization conditions. Knowledge of the degree of crystallinity and the semicrystalline morphology is important as this influences the polymer thermomechanical properties. It is well documented that, for example, the melting point is proportional to the crystal layer thickness and that Young's modulus of the material increases with increasing degree of crystallinity (Bower, 2002).

Methods and models exist to interpret the small-angle X-ray scattering (SAXS) of such systems in terms of crystalline content, average crystal and amorphous layer thickness, and even complete layer thickness distributions. Popular methods include fitting the SAXS patterns with one-dimensional paracrystalline models (Hosemann \& Bagchi, 1962) or extracting information from linear correlation (Vonk \& Kortleve, 1967; Strobl \& Schneider, 1980; Goderis et al., 1999) and interface distribution functions (Ruland, 1977) via fitting procedures or more simple graphical analyses. All these methods, including the ones based on correlation and interface 
distribution functions, rely on the shape of the SAXS pattern and yield realistic morphological parameters only when the adopted morphological model is relevant to the system at hand. The morphological model in the case of two-phase lamellar systems involves assumptions on the layer thickness distributions and on whether or not crystalline and amorphous layers with a given thickness are distributed randomly over the sample volume. The model 'relevance' can be checked by comparing the degree of crystallinity as obtained via the above-mentioned model-based SAXS approaches with the value obtained via independent crystallinity sensitive techniques, like for example wide-angle X-ray diffraction (WAXD), density measurements or calorimetric methods (Swan, 1960; Mathot, 1994). However, since each technique probes crystallinity in a different way, systematically different crystallinity estimates may be obtained, which makes such comparisons less suited for a rigorous validation of the SAXS morphological model. The necessary model validation can be made by conducting SAXS experiments on an absolute scale and by considering the SAXS total scattering power besides the shape of the scattering pattern since the SAXS total scattering power contains independent information on the material crystallinity.

Polymer semicrystalline lamellar morphologies can be considered as ideal two-phase systems. It is rarely justified to account for diffuse interfaces between the crystalline and amorphous phases since such interfaces may easily be introduced by exaggerating background corrections, in particular when SAXS is not measured up to sufficiently high angles. This has been clearly demonstrated for a sample of linear polyethylene (Goderis et al., 1999). The absolute SAXS total scattering power, $Q$, in the case of two-phase systems with sharp phase boundaries can be expressed in (electrons) ${ }^{2} \mathrm{~cm}^{-6}$ and can be written as (Porod, 1951; Roe, 2000; Strobl \& Schneider, 1980)

$$
\begin{aligned}
Q & =\frac{1}{r_{\mathrm{e}}^{2}(2 \pi)^{3}} \int_{0}^{\infty} 4 \pi q^{2}\left[\frac{\mathrm{d} \Sigma}{\mathrm{d} \Omega}(q)-B\right] \mathrm{d} q \\
& =\alpha_{\mathrm{S}}\left(\rho_{\mathrm{C}}-\rho_{\mathrm{A}}\right)^{2} \varphi_{\mathrm{L}}\left(1-\varphi_{\mathrm{L}}\right) \quad\left[(\text { e.u. })^{2} \mathrm{~cm}^{-6}\right] .
\end{aligned}
$$

Here $r_{\mathrm{e}}$ stands for the classical Thomson electron radius $\left(2.8179 \times 10^{-13} \mathrm{~cm}\right), B$ is the background due to local density fluctuations and $\mathrm{d} \Sigma / \mathrm{d} \Omega(q)$ is the absolute SAXS intensity as a function of $q$, the modulus of the momentum transfer $[q=|\mathbf{q}|=$ $(4 \pi / \lambda)(\sin \theta)$, with $\theta$ half the scattering angle and $\lambda$ the X-ray wavelength]. In this formalism it is assumed that semicrystalline regions are embedded in an amorphous or liquid-like matrix (Goderis et al., 1999). $\varphi_{\mathrm{L}}$ is the volume fraction of crystalline material within the semicrystalline regions and $\alpha_{\mathrm{S}}$ is the volume fraction of the semicrystalline regions. The subscript ' $L$ ' for $\varphi_{\mathrm{L}}$ emphasizes that this parameter represents a local crystallinity. The crystalline fraction of the entire material equals $\alpha_{\mathrm{S}} \varphi_{\mathrm{L}} \cdot \rho_{\mathrm{C}}$ and $\rho_{\mathrm{A}}$ are the electron densities of, respectively, the crystalline and amorphous phases within the semicrystalline regions (Goderis et al., 1999). The factor $\alpha_{\mathrm{S}}$ typically represents the fraction of growing micrometre-sized semicrystalline spherulites during the crystallization process or accounts for the larger amorphous pools that develop in the melting process. At the end of the crystallization process or at temperatures below the onset of bulk melting during heating, the entire volume is occupied by semicrystalline layer stacks and $\alpha_{\mathrm{S}}$ equals unity. In these cases, $\varphi_{\mathrm{L}}$ characterizes the crystalline fraction of the entire material, and with knowledge of the electron densities one can derive $\varphi_{\mathrm{L}}$ from $Q$. $Q$, sometimes referred to as the 'invariant', does not depend on the actual arrangement of the crystalline and amorphous volumes (provided $\alpha_{\mathrm{S}}=1$; Goderis et al., 1999) and therefore yields a model-independent SAXS-based $\varphi_{\mathrm{L}}$ value. This value can be compared with $\varphi_{\mathrm{L}}$ obtained from a model-based SAXS curveshape analysis, to validate the morphological model. Alternatively, $\varphi_{\mathrm{L}}$ from $Q$ can be used as a constraint within the model to obtain realistic morphologies.

This $Q$-based approach only works when values for $\rho_{\mathrm{C}}$ and $\rho_{\mathrm{A}}$ are available. $\rho_{\mathrm{C}}$ can readily be obtained from WAXD via the crystalline unit-cell dimensions and the known number of electrons within the unit cell. Some polymers with a low crystallization rate and high glass transition temperature $\left(T_{\mathrm{g}}\right)$ can be rapidly cooled into the amorphous state and consequently allow for a direct measurement of the amorphous mass density, $d_{\mathrm{A}}\left(\mathrm{g} \mathrm{cm}^{-3}\right)$, which can be converted into $\rho_{\mathrm{A}}$. This approach is, however, not possible for low $T_{\mathrm{g}}$ and rapidly crystallizing polymers such as polyethylene. As a last resort for polyethylene one often makes use of melt-extrapolated $d_{\mathrm{A}}$ values such as the ones given by Orwoll \& Flory (1967),

$$
\begin{aligned}
d_{\mathrm{A}}= & 0.8674-6.313 \times 10^{-4} T-0.367 \times 10^{-6} T^{2} \\
& -0.055 \times 10^{-8} T^{3} \quad\left[\mathrm{~g} \mathrm{~cm}^{-3}\right],
\end{aligned}
$$

or by Swan (1960),

$$
d_{\mathrm{A}}=\frac{1}{1.1360+0.885 \times 10^{-3} T} \quad\left[\mathrm{~g} \mathrm{~cm}^{-3}\right] .
$$

In equations (2) and (3) $T$ is the temperature $\left[{ }^{\circ} \mathrm{C}\right]$ (where $\left.\left[{ }^{\circ} \mathrm{C}\right]=[\mathrm{K}]-273.15\right)$.

Both the quenching and melt-extrapolation approaches take for granted that the amorphous density in between the crystallites is identical to that of glass or the pure melt. However, Swan suggested that $d_{\mathrm{A}}$ in semicrystalline highdensity polyethylene (HDPE) may well be higher than that of the extrapolated melt and that its value may depend on the sample crystallinity (Swan, 1960; Orwoll \& Flory, 1967). Up to now, these hypotheses have never been carefully checked.

In the present paper the amorphous phase density in a slowly cooled, highly crystalline HDPE sample, displaying an ideal lamellar morphology, will serve as a reference. For such systems, SAXS data processing procedures are available to reliably extract $\varphi_{\mathrm{L}}$ from the shape of the scattering patterns. With the obtained $\varphi_{\mathrm{L}}$ values and the WAXD-based crystalline density, the amorphous density will be extracted from $Q$ at a series of temperatures. Following similar procedures, the temperature-dependent amorphous density in a lamellar, rather low crystalline poly(ethylene-co-1-octene) sample will be determined to address the crystallinity dependence of the 
amorphous density in semicrystalline polyethylene. It will be demonstrated that there is none.

Finally, it will be shown that SAXS curve-shape analyses yield unrealistic $\varphi_{\mathrm{L}}$ values for a more rapidly cooled HDPE sample, because of a non-ideal layer stacking. Using the obtained $\rho_{\mathrm{C}}$ and $\rho_{\mathrm{A}}$ values, a $Q$-based $\varphi_{\mathrm{L}}$ value will be calculated and used as a constraint in a modified SAXS curve-shape analysis procedure to obtain realistic values for the average thickness of the crystalline and amorphous layers.

\section{Experimental}

\subsection{Materials and sample thermal history}

A commercial HDPE and a metallocene catalyst-based ethylene-1-octene copolymer were kindly provided by DSM Research, Geleen, The Netherlands. The HDPE has a weightaveraged molar mass of $77000 \mathrm{~g} \mathrm{~mol}^{-1}$ and a polydispersity $\left(M_{\mathrm{w}} / M_{\mathrm{n}}\right)$ of 5.9 . The ethylene copolymer with $5.5 \mathrm{~mol} \%$ of 1-octene has a weight-average molar mass of $88000 \mathrm{~g} \mathrm{~mol}^{-1}$ and a polydispersity of 2.44 and an overall density of $0.899 \mathrm{~g} \mathrm{~cm}^{-3}$ at $23^{\circ} \mathrm{C}$. Hexyl branches are appended to the linear polyethylene chain if 1-octene is used as a comonomer. Branched chain fragments cannot be incorporated into the crystalline lamellae, and consequently the crystal thickness and crystallinity decrease with increasing 1-octene content (Vonk \& Reynaers, 1990). At too high comonomer contents, the crystallites may depart from their classical lamellar habit (Bensason et al., 1996). At a comonomer content of $5.5 \mathrm{~mol} \%$ the material still consists of lamellar crystallites (Goderis et al., 2002).

Samples were brought for $5 \mathrm{~min}$ into the melt state $\left(180^{\circ} \mathrm{C}\right)$

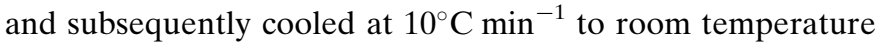
in a Mettler FP-82HT hot stage flushed with nitrogen. In addition, a sample of HDPE cooled at $1^{\circ} \mathrm{C} \mathrm{min}{ }^{-1}$ to room temperature was prepared. This sample was termed HDPE1, while the one cooled at $10^{\circ} \mathrm{C} \mathrm{min}^{-1}$ is referred to as HDPE10.

\subsection{WAXD}

The temperature-dependent crystal densities of HDPE10 and the copolymer were determined from WAXD data recorded at the Dutch-Belgian Beamline (DUBBLE-CRG, ESRF, Grenoble, France). Samples, wrapped in Al foil, were placed in a Linkam DSC600 hot stage for temperature control. Data were collected on a curved microstrip detector (Zhukov et al., 1997) over the angular range $8 \leq 2 \theta \leq 60^{\circ}$ (with $2 \theta$ the scattering angle) and using an X-ray wavelength of $1.24 \AA$. The scattering angles were calibrated by using the HDPE orthorhombic 110 and 200 reflections collected previously at room temperature using a horizontal Geigerflex diffractometer on a Rigaku RU-200B rotating $\mathrm{Cu}$ anode, calibrated with a standard NBS silicon powder. Specimens from the same set of samples as in the SAXS experiments were used. After being cooled to $-30^{\circ} \mathrm{C}$, the samples were heated continuously at $10^{\circ} \mathrm{C} \mathrm{m^{-1 }}$. Data were collected in consecutive time frames of $6 \mathrm{~s}$, corresponding to a temperature resolution of $1^{\circ} \mathrm{C}$. A background was subtracted from the data after correction for the detector response and normalization to the intensity of the primary beam, measured by an ionization chamber placed downstream from the sample. The scattering patterns at temperatures corresponding to the SAXS experiments were selected for further processing.

A linear background was subtracted from the patterns, which were subsequently decomposed into the 110 and 200 reflections (both modeled by a Pearson-type function) and the amorphous halo (modeled by a Lorentz function). The Marquardt-Levenberg nonlinear least-squares curve-fitting algorithm, as implemented in Microcal Origin (Version 6.0, Microcal Software Inc., Northampton, MA, USA), was used. The crystalline density was calculated from the angular positions of the Pearson maxima of the 110 and 200 reflections, assuming that the length of the orthorhombic unit cell in the $c$ direction is constant and equal to $2.547 \AA$.

\subsection{SAXS}

The Bruker NanoSTAR instrument located in the Chemistry Department of the University of Aarhus Denmark (Pedersen, 2004) was used for the SAXS measurements. Data were registered in the range $0.0071 \leq q \leq 0.3341 \AA^{-1}$. To control the sample temperature during the measurement, an Anton Paar KPR cooling/heating unit was used. The samples were cooled from room temperature to $-30^{\circ} \mathrm{C}$ at $10^{\circ} \mathrm{C} \mathrm{min}{ }^{-1}$ in the camera, and then a stepwise heating program was applied. After $1 \mathrm{~h}$ of equilibration at a given temperature, a scattering pattern was recorded for $1800 \mathrm{~s}$, after which the sample was heated $10^{\circ} \mathrm{C}$ higher at $10^{\circ} \mathrm{C} \mathrm{min}^{-1}$ for equilibration, followed by another measurement and a next temperature increase. Both HDPE samples were heated to $120^{\circ} \mathrm{C}$, while the copolymer was heated only to $60^{\circ} \mathrm{C}$. The HDPE melts in bulk at $134^{\circ} \mathrm{C}$, while the copolymer does so at $95^{\circ} \mathrm{C}$. Limiting the measurements to the upper temperatures ensures that $\alpha_{\mathrm{S}}$ equals 1 in all cases. This facilitates the analysis (Goderis et al., 2002).

The sample transmission, $T_{\mathrm{S}}$, at each temperature was measured by collecting the strong scattering of glassy carbon, mounted in the beam path downstream from the sample, and using

$$
T_{\mathrm{S}}=\left(I_{\mathrm{GC}+\mathrm{S}}-T_{\mathrm{GC}} I_{\mathrm{S}}\right) / I_{\mathrm{GC}}
$$

where $I_{\mathrm{GC}}$ and $I_{\mathrm{S}}$ are the integrated scattering intensity of glassy carbon and of the sample measured separately, while $I_{\mathrm{GC}+\mathrm{S}}$ is the integrated scattering of glassy carbon measured in row with the sample. $T_{\mathrm{GC}}$ is the known glassy carbon transmission. All intensities were corrected for the detector dark current prior to being used in equation (4).

The two-dimensional powder patterns were azimuthally averaged using the Bruker SAXS software and corrected for beamstop shadowing (Pedersen, 2004). Next, the isotropic scattering patterns were normalized to the acquisition time and the incident beam intensity, monitored by a piece of lowdensity polyethylene, yielding $I_{\text {nor }}(q)$. The background was subtracted from $I_{\text {nor }}(q)$ taking into account transmissions and dark current signals, resulting in $I(q)$ : 


$$
I(q)=\frac{1}{T_{\mathrm{S}}}\left[I_{\mathrm{nor}}(q)-I_{\mathrm{nor}}^{\mathrm{dark}}(q)\right]-\frac{1}{T_{\mathrm{BG}}}\left[I_{\mathrm{nor}}^{\mathrm{BG}}(q)-I_{\mathrm{nor}}^{\mathrm{dark}}(q)\right],
$$

where $I_{\text {nor }}^{\mathrm{BG}}(q)$ is the normalized background intensity. $T_{\mathrm{S}}$ and $T_{\mathrm{BG}}$ are sample and background transmissions and $I_{\text {nor }}^{\mathrm{dark}}(q)$ is the detector dark current normalized to its accumulation time.

Finally, $I(q)$ was scaled to absolute intensities, $\mathrm{d} \Sigma / \mathrm{d} \Omega(q)$, using the known scattering intensity of water at $q=0$ as a primary standard (Pedersen, 2004), i.e. $0.0164 \mathrm{~cm}^{-1}$ at $20^{\circ} \mathrm{C}$ :

$$
\frac{\mathrm{d} \Sigma}{\mathrm{d} \Omega}(q)=\left[\frac{I(q) l_{\text {water }}}{I_{\text {water }}(0) l_{\text {sample }}}\right] 0.0164 \quad\left[\mathrm{~cm}^{-1}\right] .
$$

In equation (6), $I_{\text {water }}(0)$ represents the measured scattering intensity of water, linearly extrapolated to $q=0 . l_{\text {water }}$ and $l_{\text {sample }}$ are the thicknesses of water and the sample, respectively $[\mathrm{cm}] . l_{\text {sample }}$ was measured using a caliper, while $l_{\text {water }}$ was determined from Lambert's law:

$$
T_{\text {water }}=\exp \left(-\mu_{\text {water }} l_{\text {water }}\right),
$$

with $\mu_{\text {water }}=0.1 \mathrm{~cm}^{-1}$ the linear absorption coefficient of water and $T_{\text {water }}$ the measured water transmission.

All SAXS patterns were analyzed in terms of a model that assumes powders of one-dimensional stacks of alternating, laterally extended crystalline and amorphous layers, the electron density profile of which is sketched in Fig. 1 (gray line). Such a morphology implies that the average crystalline layer thickness, $\left\langle l_{\mathrm{C}}\right\rangle$, is given by

$$
\left\langle l_{\mathrm{C}}\right\rangle=\varphi_{\mathrm{L}}\left\langle L_{\mathrm{P}}\right\rangle
$$

and the average amorphous layer thickness, $\left\langle l_{\mathrm{A}}\right\rangle$, by

$$
\left\langle l_{\mathrm{A}}\right\rangle=\left(1-\varphi_{\mathrm{L}}\right)\left\langle L_{\mathrm{P}}\right\rangle,
$$

with $\left\langle L_{\mathrm{P}}\right\rangle$ the number-average repeat distance, often referred to as the 'long period'. The most probable crystalline and

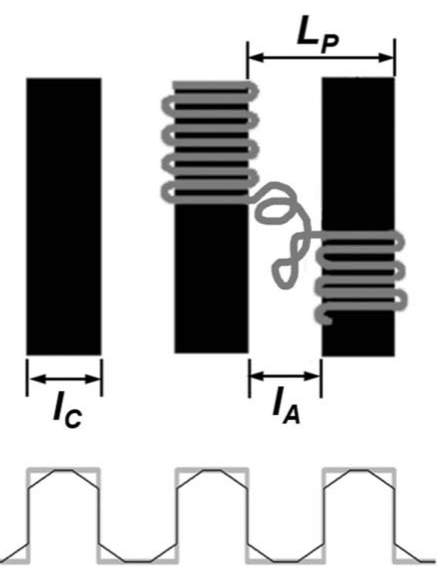

Figure 1

Electron density profiles for a one-dimensional stack of alternating crystalline (electron density $\rho_{\mathrm{C}}$ ) and amorphous layers (electron density $\left.\rho_{\mathrm{A}}\right)$ with most probable thickness $l_{\mathrm{C}}$ and $l_{\mathrm{A}}$, respectively. The most probable repeat distance or 'long period' between two identical units equals $L_{\mathrm{P}}$. The ideal two-phase profile assumed in the analysis of SAXS data is depicted in gray. The electron density profile relevant to the ethylene copolymers is represented by a black line. Both profiles display sharp interfaces, justifying the applicability of Porod's law. amorphous layer thicknesses are represented by $l_{\mathrm{A}}$ and $l_{\mathrm{C}}$, the most probable repeat distance by $L_{\mathrm{P}}$.

The SAXS curve-shape analysis was done by means of linear correlation functions, $K(x)$, describing the spatial correlation of the electron density fluctuations in a direction, $x$, normal to the lamellar surfaces and by means of interface distribution functions, $\operatorname{IDF}(x)$, representing the probability of finding two interfaces at a certain distance $x$ from each other (Ruland, 1977). $\operatorname{IDF}(x)$ can be considered as the second derivative (curvature) of $K(x) . K(x)$ is the Fourier transform of the scattering intensity and was calculated as (Strobl \& Schneider, 1980)

$$
K(x)=\frac{1}{r_{\mathrm{e}}^{2}(2 \pi)^{3}} \int_{0}^{\infty}\left\{\left[\frac{\mathrm{d} \Sigma}{\mathrm{d} \Omega}(q)-B\right] 4 \pi q^{2} \cos (q x)\right\} \mathrm{d} q,
$$

with $B$ a constant, representing the background due to local density fluctuations. Prior to applying equation (10), $[\mathrm{d} \Sigma /$ $\mathrm{d} \Omega(q)-B]$ was extrapolated to high and zero $q$ values to avoid truncation errors. The extrapolation to $q=0$ is uncritical because it is minimized by the $q^{2}$ factor in equation (10), and the value at $q=0$ was taken to be equal to the value at the lowest experimental data point, i.e. at $q=0.0071 \AA^{-1}$. To extrapolate the scattering intensity to high $q$, Porod's law, $[\mathrm{d} \Sigma /$ $\mathrm{d} \Omega(q)-B]=P / q^{4}$, was used (Porod, 1951), where $P$ is the Porod constant, thereby implicitly assuming that the boundaries between the crystalline and amorphous regions are sharp. Suitable values for $B$ and $P$ were found by calculating the interference function $G(q)$,

$$
G(q)=\left[\frac{\mathrm{d} \Sigma}{\mathrm{d} \Omega}(q)-B\right]\left(\frac{q}{2 \pi}\right)^{4}-P,
$$

while obeying the following two constraints (Albrecht \& Strobl, 1995):

$$
\begin{aligned}
& \lim _{q \rightarrow \infty} G(q)=0, \\
& \int_{0}^{\infty} G(q) \mathrm{d} q=0 .
\end{aligned}
$$

In the minimization procedure to find $B$ and $P$, data were truncated at $q=0.273 \AA^{-1}$ to avoid solutions for $B$ and $P$ that are biased by $\mathrm{d} \Sigma / \mathrm{d} \Omega(q)$ data with poor counting statistics at higher $q$ values. The value at the origin of $K(x), K(0)$, equals $Q$ as defined by equation (1). Recall that, because measurements are not taken at very high temperatures, $\alpha_{\mathrm{S}}$ equals 1 in all considered cases. Dividing $K(x)$ by $Q$ yields the normalized correlation function $\mathrm{CF}(x)$ (Goderis et al., 1999). $\operatorname{IDF}(x)$ was calculated by Fourier transformation of $\mathrm{G}(q)$ (Ruland, 1977):

$$
\operatorname{IDF}(x)=-\frac{2}{r_{\mathrm{e}}^{2}(2 \pi)^{2}} \int_{0}^{\infty} G(q) \cos (q x) \mathrm{d} q .
$$


Table 1

SAXS number-average morphological parameters and (volume fraction) crystallinities, $\varphi_{\mathrm{L}}$.

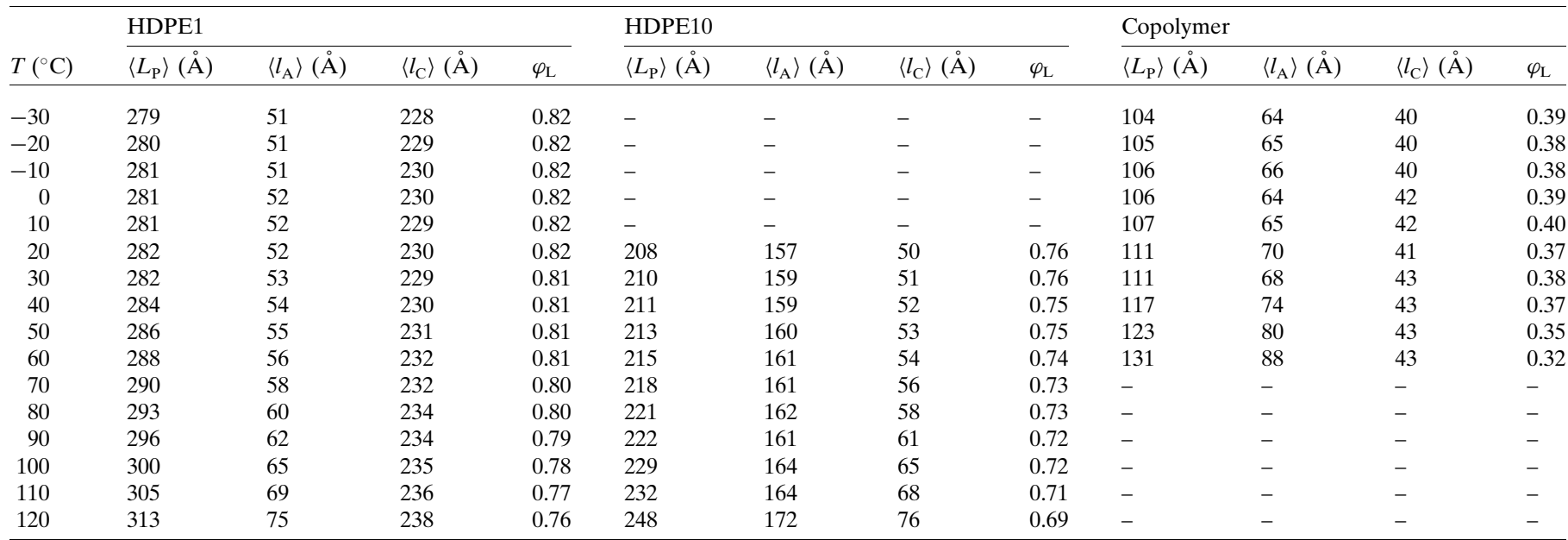

\section{Results and discussion}

3.1. Extracting morphological parameters from the SAXS curve shape: HDPE1 and the copolymer

Fig. 2 illustrates the absolute SAXS patterns at $-30^{\circ} \mathrm{C}$ for the three samples studied. In Fig. 3, $\mathrm{CF}(x)$ of HDPE1 is shown together with the linear regression to the autocorrelation triangle (gray dashed line), which for a two-phase onedimensional stack of laterally extended layers is given by (Goderis et al., 1999)

$$
y=-\frac{1}{\left\langle L_{\mathrm{P}}\right\rangle \varphi(1-\varphi)} x+1
$$

with $\varphi$ the minority fraction within the semicrystalline layer stacks. A flat $\mathrm{CF}(x)$ baseline, of which the $\mathrm{CF}(x)$ baseline in Fig. 3 is an example, is observed if the amorphous and crystalline layer thickness distributions do not overlap. This baseline, which will be referred to as $\mathrm{CF}_{\text {min }}$, is given by (Vonk \& Kortleve, 1967; Strobl \& Schneider, 1980; Goderis et al., 1999)



Figure 2

Absolute SAXS intensities of the three samples recorded at $-30^{\circ} \mathrm{C}$.

$$
\mathrm{CF}_{\text {min }}=\frac{-\varphi}{(1-\varphi)}
$$

Combining equation (15) with equation (16) in which $y=$ $\mathrm{CF}_{\text {min }}$ yields the intersection of the linear regression with the $\mathrm{CF}(x)$ baseline at an $x$ value given by

$$
x=\varphi\left\langle L_{\mathrm{P}}\right\rangle \text {. }
$$

As $\varphi$ is the amorphous phase in HDPE, it follows that $\varphi=(1-$ $\left.\varphi_{\mathrm{L}}\right)$ and from equation (9) that the intersection occurs at $x=$ $\left\langle l_{\mathrm{A}}\right\rangle$, as indicated in Fig. 3. It also follows that $\varphi_{\mathrm{L}}$ can be retrieved from equation (16), using $\mathrm{CF}_{\min }$ as input with $\varphi=$ $\left(1-\varphi_{\mathrm{L}}\right)$. Since $\left\langle l_{\mathrm{A}}\right\rangle$ and $\varphi_{\mathrm{L}}$ are known, also $\left\langle L_{\mathrm{P}}\right\rangle$ and $\left\langle l_{\mathrm{C}}\right\rangle$ are readily obtained from equations (9) and (8), respectively. The as obtained HDPE1 morphological parameters for all studied temperatures are listed in Table 1.

Note that $\left\langle L_{\mathrm{P}}\right\rangle$ is systematically lower than $L_{\mathrm{CF}}$, the second maximum in $\mathrm{CF}(x)$, but that $\left\langle L_{\mathrm{P}}\right\rangle$ corresponds rather well to

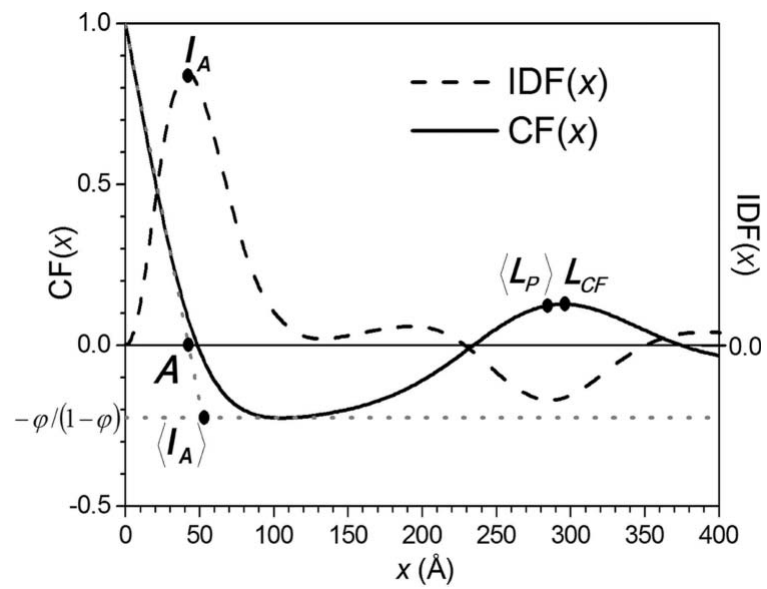

Figure 3

$\operatorname{IDF}(x)$ (dashed line) and $\mathrm{CF}(x)$ (solid line) of HDPE1 at $20^{\circ} \mathrm{C}$. The intersection of the linear regression to the autocorrelation triangle of $\mathrm{CF}(x)$ (dashed gray line) with the horizontal dotted gray line at $-\varphi /(1-$ $\varphi)$ yields $\left\langle l_{\mathrm{A}}\right\rangle$. The first maximum of the interface distribution function corresponds to the most probable amorphous layer thickness $l_{\mathrm{A}}$, which in this case nearly equals $\left\langle l_{\mathrm{A}}\right\rangle$. 
the minimum in $\operatorname{IDF}(x)$ (Fig. 3). For lamellar two-phase systems with $\varphi_{\mathrm{L}}>0.5$, the first two positive peaks in $\operatorname{IDF}(x)$ represent the thickness distribution of the amorphous and crystalline layer, respectively, whereas the first negative contribution at higher $x$ is related to the thickness distribution of the long period (Ruland, 1977). In principle, the maxima in these distributions can be considered as most probable values, $l_{\mathrm{A}}, l_{\mathrm{C}}$ and $L_{\mathrm{P}}$. With $\left\langle L_{\mathrm{P}}\right\rangle$ corresponding rather well to $L_{\mathrm{P}}$ it appears that the long period thickness distribution is symmetric. This also holds for the amorphous layer thickness distribution, since the peak in $\operatorname{IDF}(x), l_{\mathrm{A}}$, corresponds well to $\left\langle l_{\mathrm{A}}\right\rangle$. For HDPE1, $l_{\mathrm{C}}$ cannot be determined, since its distribution strongly overlaps with and partially annihilates the left wing of the negative long period distribution. However, given the symmetry of the amorphous layer and periodicity distribution, the thickness distribution of the crystalline layers should also be symmetric. Crist (2000) explicitly demonstrated, on the basis of an analysis of model SAXS patterns, that the position of the minimum in $\operatorname{IDF}(x)$ equals $\left\langle L_{\mathrm{P}}\right\rangle$ for symmetric distributions and that $\left\langle L_{\mathrm{P}}\right\rangle$ in that case is systematically lower than $L_{\mathrm{CF}}$ when the reduced standard deviation of the periodicity exceeds a given value. In fact, the graphs in Fig. 3 are identical to the model calculations by Crist with $\varphi_{\mathrm{L}}=$ 0.8 and a reduced standard deviation of the symmetric periodicity distribution of 0.2 [see Fig. 3 of Crist (2000)]. As the $\left\langle L_{\mathrm{P}}\right\rangle$ value calculated from $\mathrm{CF}(x)$ is equal to the (correct) one from $\operatorname{IDF}(x)$ (as all distributions appear to be symmetric) it is safe to state that also the $\mathrm{CF}(x)$-based crystallinity is correct. This statement is supported by the observation that $\operatorname{IDF}(x)$ of HDPE1 in the region $100<x<150 \AA$ passes a minimum close to zero, indicating that the amorphous and crystalline layer distributions do not overlap, which is a prerequisite for using equation (16).

When the layer thickness distributions overlap, the $\mathrm{CF}(x)$ baseline is not flat and the $-\varphi /(1-\varphi)$ level falls below $\mathrm{CF}_{\min }$ (Strobl \& Schneider, 1980). The single positive maximum in the copolymer $\operatorname{IDF}(x)$ demonstrates that the crystalline and amorphous layer thickness distributions indeed heavily

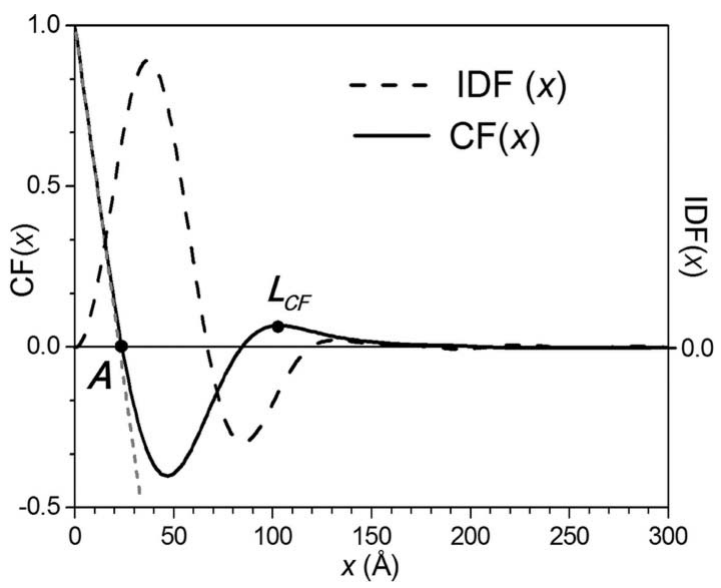

Figure 4

$\mathrm{CF}(x)$ (dashed line) and $\operatorname{IDF}(x)$ (solid line) of the copolymer at $-30^{\circ} \mathrm{C}$. The linear regression to the autocorrelation triangle of $\mathrm{CF}(x)$ is represented by a dashed gray line. overlap (Fig. 4). For such systems $\varphi$ cannot be obtained from $\mathrm{CF}_{\text {min }}$ and equation (16). Solving equation (15) for $y=0$ yields the so-called quadratic expression (Goderis et al., 1999):

$$
A=\varphi(1-\varphi)\left\langle L_{\mathrm{P}}\right\rangle
$$

with $A$ the intersection of the linear regression to the autocorrelation triangle with the abscissa, indicated in Figs. 3 and 4 for HDPE1 and the copolymer, respectively. In favorable cases, $L_{\mathrm{CF}}$ can be used as input instead of $\left\langle L_{\mathrm{P}}\right\rangle$ together with $A$, to solve equation (18) for $\varphi$. Obviously, solving equation (18) brings two results, of which the lowest corresponds to $\varphi$ and the highest to $(1-\varphi)$. Equation (18) was used to obtain $\varphi$ for the copolymer, assuming that $\left\langle L_{\mathrm{P}}\right\rangle$ equals $L_{\mathrm{CF}}$. Support for this assumption will be provided in $\$ 3.2$. Since we know that $\varphi$ corresponds to $\left(1-\varphi_{\mathrm{L}}\right)$ for this low-crystalline material (Goderis et al., 2002), $\left\langle l_{\mathrm{A}}\right\rangle$ and $\left\langle l_{\mathrm{C}}\right\rangle$ can readily be calculated using equations (8) and (9). All copolymer morphological data obtained via this approach are listed in Table 1.

\subsection{Extracting amorphous densities from absolute $Q$ values:} HDPE1 and the copolymer

The crystalline mass density, $d_{\mathrm{C}}$, was calculated from the WAXD crystalline unit cell and the known unit cell mass for polyethylene. The HDPE1 WAXD-based crystalline density values (not shown) agree well with the densities given earlier by Swan (1960):

$$
d_{\mathrm{C}}=\frac{1}{0.994+2.614 \times 10^{-4} T+4.43 \times 10^{-7} T^{2}} .
$$

In equation (19) the temperature, $T$, is expressed in ${ }^{\circ} \mathrm{C}$. The Swan crystalline density line was therefore assumed to adequately represent the HDPE1 crystalline density. $\rho_{\mathrm{C}}$ was calculated from $d_{\mathrm{C}}$ by multiplying the $d_{\mathrm{C}}$ values by $\left(N_{\mathrm{A}} \times 8 /\right.$ 14) [(e.u.) $\mathrm{g}^{-1}$ ], with $N_{\mathrm{A}}$ Avogadro's number and 8/14 the ratio of the number of electrons to the molar mass of the repeat unit in polyethylene. Using these $\rho_{\mathrm{C}}$ values, $\rho_{\mathrm{A}}$ values were calculated for HDPE1 using

$$
\rho_{\mathrm{A}}=\left\{\rho_{\mathrm{C}}-\left[\frac{Q}{\varphi_{\mathrm{L}}\left(1-\varphi_{\mathrm{L}}\right)}\right]^{1 / 2}\right\} \quad\left[\text { e.u. } \mathrm{cm}^{-3}\right] .
$$

The $\rho_{\mathrm{A}}$ values from equation (20) were converted to massbased $d_{\mathrm{A}}$ values by multiplying with $\left(N_{\mathrm{A}}{ }^{-1} \times 14 / 8\right)$ [g (e.u. $)^{-1}$ ]. The calculations were limited to temperatures above the glass transition temperature range of the amorphous phase, which for semicrystalline $\mathrm{HDPE}$ is from -153 to $17^{\circ} \mathrm{C}$ (Mathot, 1986). The results are illustrated in Fig. 5. The $d_{\mathrm{A}}$ value of amorphous (liquid) material enclosed between crystallites seems to be slightly lower than that of the HDPE melt, in contrast to what has been suggested earlier (Swan, 1960; Orwoll \& Flory, 1967).

An identical procedure was applied to the copolymer. The results are included in Fig. 5 down to $-35^{\circ} \mathrm{C}$, since vitrification for a copolymer with $5.5 \mathrm{~mol} \%$ 1-octene occurs below that temperature (Vanden Eynde et al., 2000). The WAXD-based copolymer $d_{\mathrm{C}}$ and the $d_{\mathrm{A}}$ values both fall below the HDPE1 values. However, if the Swan crystalline density values are 
used in equation (20) instead of the WAXD copolymer values, amorphous densities (filled diamonds in Fig. 5) are obtained that coincide with the HDPE values (open squares). Below, the thesis is defended that the HDPE crystalline densities apply to the copolymer, rather than its own WAXD-based values.

With the SAXS-based copolymer $\varphi_{\mathrm{L}}$ value and the HDPE amorphous and crystalline densities at $20^{\circ} \mathrm{C}$ an overall copolymer density can be calculated which is very close to that reported by the manufacturer at $23^{\circ} \mathrm{C}$ (see Experimental section). With the WAXD-based copolymer $d_{\mathrm{C}}$, too low values are obtained. Secondly, with the WAXD copolymer crystalline densities it would seem that both $d_{\mathrm{C}}$ and $d_{\mathrm{A}}$ are crystallinity dependent. However, if this were true, one would expect the copolymer $d_{\mathrm{A}}$ values to lie in between the extrapolated melt and semicrystalline HDPE values, not below the HDPE values.

Accepting that the HDPE $d_{\mathrm{C}}$ values are relevant for the copolymer, an explanation is needed for the lower WAXDbased copolymer $d_{\mathrm{C}}$ values. The HDPE1 and copolymer WAXD patterns at $-30^{\circ} \mathrm{C}$ are illustrated in Fig. 6. The maxima of the copolymer orthorhombic 110 and 200 reflections occur at lower angles, are broader and are less intense compared to in HDPE1. However, the high-angle shoulders of the copolymer crystalline peaks coincide with those of HDPE1, pointing to a spread of crystal unit-cell sizes with an important fraction of high-density HDPE-like unit cells besides more open ones diffracting at lower angles (Basiura et al., 2006). In Fig. 1, a plausible copolymer electron density profile is sketched, as suggested earlier by Strobl \& Schneider (1980). The centers of the crystals are defect free and reach the HDPE reference crystalline density, $\rho_{\mathrm{C}}$. The unit cells at the crystal borders are expanded owing to local stress and chain conformational defects as a result of comonomer crowding. One can also expect a local densification of the

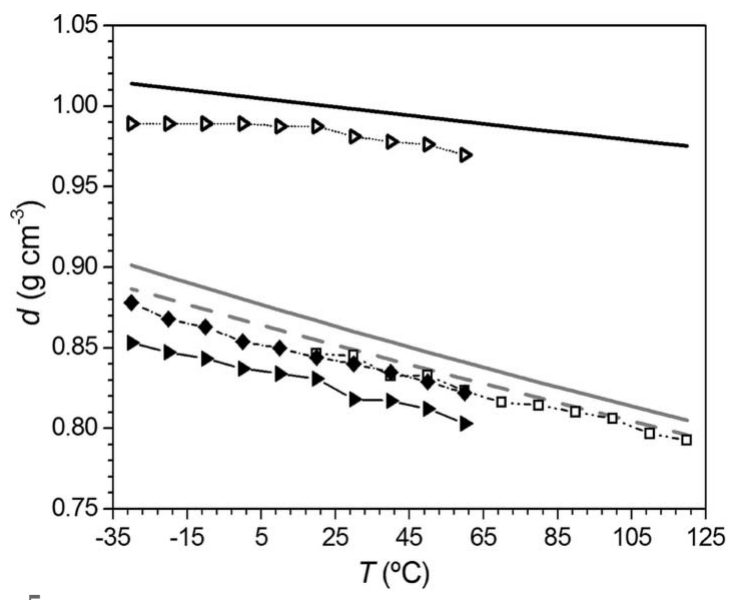

Figure 5

Phase densities. Full black line: Swan crystalline density line (representing the HDPE crystalline density); WAXD-based crystalline density of the copolymer (open triangles); calculated amorphous density of HDPE1 (empty squares), copolymer (filled triangles) and copolymer with the assumption that the copolymer crystalline density is identical to that of HDPE (filled diamonds). Gray solid line: Swan amorphous density line; gray dashed line: Orwoll-Flory amorphous density line. amorphous regions close to the crystals due to a crowding of comonomer units. When the SAXS patterns of such symmetric profiles are treated in terms of ideal two-phase systems (the gray line in Fig. 1), the crystalline core $\left(\rho_{\mathrm{C}}\right)$ and amorphous core $\left(\rho_{\mathrm{A}}\right)$ densities are relevant. Such a perfect density symmetry is probably not realistic, but slight deviations are not expected to alter the case. In Fig. 1, the fraction of the crystals with a lower density is larger than the share of their dense cores and therefore dominates the crystal peak positions in WAXD. This results in a WAXD crystalline density lower than the reference HDPE-like density. This view is in line with the widely accepted WAXD-based observation that the crystalline density of random ethylene copolymers decreases with increasing comonomer content (Clas et al., 1988; Peeters et al., 1997). This decrease seems to be related to the decreasing average crystal thickness, i.e. a reduction of the HDPE-like crystalline core (Androsch \& Wunderlich, 2000; Vonk \& Pijpers, 1985; Mandelkern, 1992) and its decreasing contribution to the WAXD patterns. The absolute SAXS results support the idea that the crystalline core of such copolymers still contains orthorhombic unit cells that are as dense as in HDPE. On these grounds, one can conclude that the crystalline and the amorphous densities of semicrystalline polyethylene, relevant to SAXS, are crystallinity independent. Furthermore, it seems that both HDPE and the copolymer reach a common amorphous density that is lower than that of a pure melt and which can be described by the following second-order polynomial:

$$
d_{\mathrm{A}}=857.7 \times 10^{-3}-6.2 \times 10^{-4} T+5 \times 10^{-7} T^{2} \quad\left[\mathrm{~g} \mathrm{~cm}^{-3}\right] .
$$

$T$ is the temperature in ${ }^{\circ} \mathrm{C}$. The second-order fitting was performed using both the HDPE1 and copolymer $d_{\mathrm{A}}$ values. These $d_{\mathrm{A}}$ values approach the Orwoll-Flory line at high temperatures but deviate from the Swan line for liquid polyethylene [equation (3)] at all temperatures. The lower density of liquid material in between crystallites suggests a lower local pressure, similar to the reduced pressure reported in the space

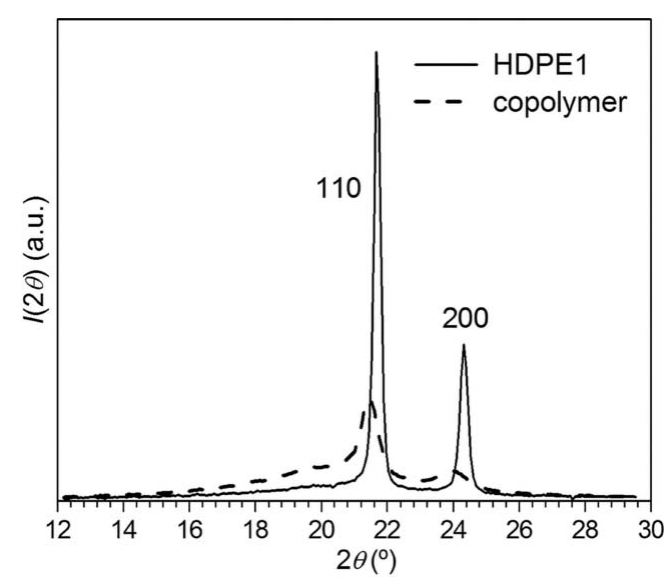

Figure 6

WAXD data recorded at $-30^{\circ} \mathrm{C}$. Note that the high-angle shoulders of the copolymer crystalline peaks coincide with those of HDPE1. 
between growing spherulites as a result of crystallizationinduced material contraction (Piorkowska \& Galeski, 1993; Varga \& Ehrenstein, 1996).

\subsection{Obtaining $\varphi_{\mathrm{L}}$ values and realistic morphological para-} meters from absolute $Q$ values and the SAXS curve shape: HDPE10

Fig. 7 displays $\mathrm{CF}(x)$ and $\operatorname{IDF}(x)$ for HDPE10. This sample does not have a flat $\mathrm{CF}(x)$ baseline, precluding the use of equation (16) for obtaining $\varphi$. Using equation (18) with $L_{\mathrm{CF}}$ as input yields the $\varphi_{\mathrm{L}}$ values as illustrated in Fig. 8. For HDPE10 too, the minority fraction is the amorphous one, hence $\varphi_{\mathrm{L}}=1-$ $\varphi$. For overlapping amorphous and crystalline layer thickness distributions, the baseline at $-\varphi /(1-\varphi)$ should occur below $\mathrm{CF}_{\text {min }}$ (Goderis et al., 1999), i.e. at a value below -0.26 for the situation at $20^{\circ} \mathrm{C}$ in Fig. 7. With $\varphi=0.18$ at $20^{\circ} \mathrm{C},-\varphi /(1-\varphi)$ equals -0.22 , which is higher than -0.26 . This inconsistency reveals that $L_{\mathrm{CF}}$ is not representative for $\left\langle L_{\mathrm{P}}\right\rangle$ and that accordingly equation (18) cannot be used with $L_{\mathrm{CF}}$ as input. Such effects are commonly encountered when the layer thickness distributions are rather broad (Crist, 2000). In the present case this may be a consequence of the rather high HDPE molar mass and the broad molar mass distribution. A fractionation of the material according to molar mass during crystallization may result in broad layer thickness distributions. In contrast, for a linear polyethylene with low molar mass and low polydispersity it was found that $L_{\mathrm{CF}}$ represents $\left\langle L_{\mathrm{P}}\right\rangle$ (Goderis et al., 1999). This problem also exists for HDPE1 ( $L_{\mathrm{CF}}$ differs from $\left\langle L_{\mathrm{P}}\right\rangle$; see Fig. 3), but in contrast to the case for HDPE10, the crystalline and amorphous layer thicknesses are well separated such that equation (16) can be used as an alternative to equation (18) with $L_{\mathrm{CF}}$.

Since neither equation (16) nor equation (18) can be used for HDPE10, an alternative can be found in a procedure that makes use of $Q$. With $\alpha_{\mathrm{S}}$ being equal to 1 and making use of

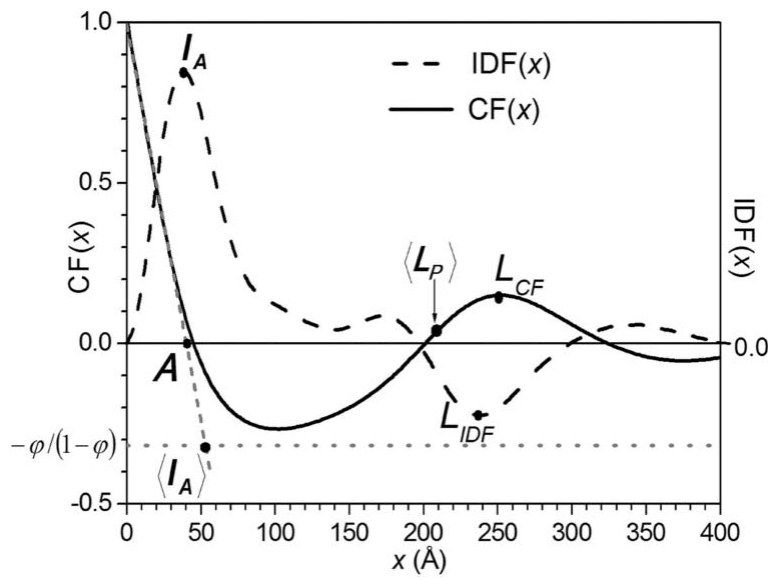

Figure 7

$\operatorname{IDF}(x)$ (dashed line) and $\mathrm{CF}(x)$ (solid line) of HDPE10 at $20^{\circ} \mathrm{C}$. The intersection of the linear regression to the autocorrelation triangle of $\mathrm{CF}(x)$ with the horizontal dotted gray line at $-\varphi(1-\varphi)$ yields $\left\langle l_{\mathrm{A}}\right\rangle$. The number-average long period, as indicated by the arrow, is smaller than the first side maximum of $\mathrm{CF}(x)$ or minimum of $\operatorname{IDF}(x)$. equations (21) and (19) for $d_{\mathrm{A}}$ and $d_{\mathrm{C}}$, followed by a conversion to $\rho_{\mathrm{A}}$ and $\rho_{\mathrm{C}}$, equation (22) was used to calculate $\varphi_{\mathrm{L}}$ :

$$
Q /\left(\rho_{\mathrm{C}}-\rho_{\mathrm{A}}\right)=\varphi_{\mathrm{L}}\left(1-\varphi_{\mathrm{L}}\right) .
$$

Since equation (22) is quadratic in $\varphi_{\mathrm{L}}$ and $\varphi_{\mathrm{L}}$ is the majority fraction in HDPE10, the solution with the highest value corresponds to $\varphi_{\mathrm{L}}$, the lowest to $\left(1-\varphi_{\mathrm{L}}\right)$. The as obtained $\varphi_{\mathrm{L}}$ values are included in Fig. 8 (open circles). Calculations were limited to temperatures above $17^{\circ} \mathrm{C}$ because at lower temperatures $d_{\mathrm{A}}$ can no longer be represented by equation (21) owing to vitrification.

Knowing the actual HDPE10 $\varphi_{\mathrm{L}}$ values (and hence also the $\varphi$ values), one can calculate $\left\langle L_{\mathrm{P}}\right\rangle$ using equation (23), which is a reshuffle of the quadratic expression, equation (18):

$$
\left\langle L_{\mathrm{P}}\right\rangle=\frac{A}{\varphi(1-\varphi)} .
$$

The product $\varphi_{\mathrm{L}}\left\langle L_{\mathrm{P}}\right\rangle$ yields $\left\langle l_{\mathrm{C}}\right\rangle$, and $\left(1-\varphi_{\mathrm{L}}\right)\left\langle L_{\mathrm{P}}\right\rangle$ yields $\left\langle l_{\mathrm{A}}\right\rangle$. The values are reported in Table $1 .\left\langle l_{\mathrm{A}}\right\rangle$ is situated at the intersection of the linear regression to the autocorrelation triangle with the baseline at $-\varphi(1-\varphi)$ in $\mathrm{CF}(x)$ as illustrated in Fig. 7. Clearly, this baseline occurs below $\mathrm{CF}_{\min }$ as it should for overlapping crystalline and amorphous layer thickness distributions. Fig. 7 also displays the correct $\left\langle L_{\mathrm{P}}\right\rangle$ value, which is clearly different from the maxima in $\mathrm{CF}(x)$ or $\operatorname{IDF}(x)$, respectively, $L_{\mathrm{CF}}$ and $L_{\mathrm{IDF}}$.

When applying the $Q$-based approach outlined above to the copolymer, $\left\langle L_{\mathrm{P}}\right\rangle$ values are obtained which coincide with the $L_{\mathrm{CF}}$ values, justifying the use of equation (18) to find $\varphi$ with $L_{\mathrm{CF}}$ as input instead of $\left\langle L_{\mathrm{P}}\right\rangle$.

\section{Conclusions}

The temperature-dependent density of the liquid-like amorphous phase enclosed between crystalline layers in semicrystalline polyethylene is crystallinity independent and is lower than melt-extrapolated values reported earlier in the literature. The amorphous densities of the investigated copolymer and HDPE samples are described by a common line.

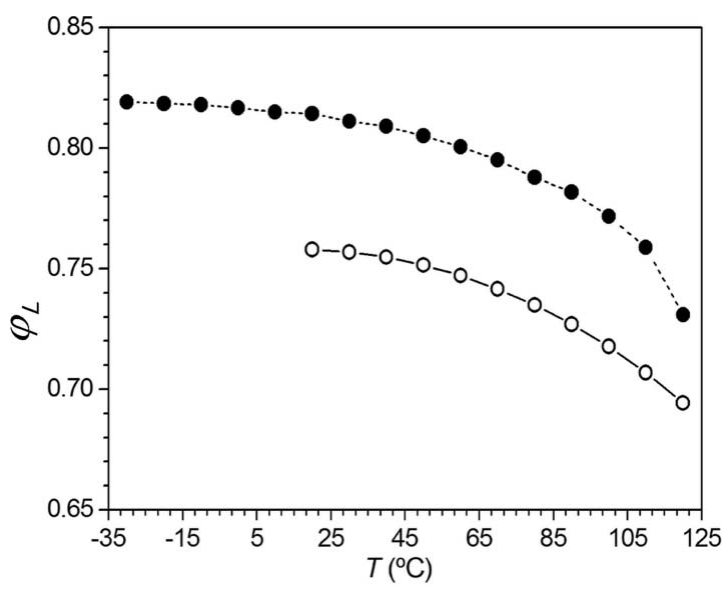

Figure 8

$\varphi_{\mathrm{L}}$ of HDPE10 based on equation (18) and using $L_{\mathrm{CF}}$ as input (filled circles); $\varphi_{\mathrm{L}}$ calculated from equation (23) (empty circles). 
The WAXD-based copolymer crystalline density is below that of HDPE owing to an important contribution of less dense orthorhombic interfacial material. The crystalline core, however, remains perfect with a density identical to that of HDPE.

Model-independent SAXS-based crystallinity values can be calculated with the aid of the densities of the crystalline and amorphous phases and the absolute SAXS invariant. It was demonstrated that such model-independent crystallinity values can be used as input in a modified SAXS curve-shape analysis procedure to obtain the average thickness of the crystalline and amorphous layers also in the case of non-ideal lamellar semicrystalline polymer morphologies for which the number-average long period cannot be retrieved from the maxima in correlation functions or interface distribution functions.

Finally, since the density values of the amorphous and crystalline phases in semicrystalline polyethylene are available now, one can safely calculate the crystallinity from its overall density.

\section{Acknowledgements}

MBC and BG thank FWO-Vlaanderen for a research grant and for funding the DUBBLE project. Harry Reynaers is acknowledged for his continuous support.

\section{References}

Albrecht, T. \& Strobl, G. (1995). Macromolecules, 28, 5267-5273.

Androsch, R. \& Wunderlich, B. (2000). Macromolecules, 33, 9076 9089.

Basiura, M., Gearba, R. I., Ivanov, D. A., Janicki, J., Reynaers, H., Groeninckx, G., Bras, W. \& Goderis, B. (2006). Macromolecules, 39, 8399-8411.
Bensason, S., Minick, J., Moet, A., Chum, S., Hiltner, A. \& Baer, E. (1996). J. Polym. Sci. B Polym. Phys. 34, 1301-1315.

Bower, D. I. (2002). An Introduction to Polymer Physics. New York: Cambridge University Press.

Clas, S. D., Heyding, R. D., McFaddin, D. C., Russell, K. E., ScammellBullock, M. V., Kelusky, E. C. \& St-Cyr, D. (1988). J. Polym. Sci. B Polym. Phys. 26, 1271-1286.

Crist, B. (2000). J. Macromol. Sci. Phys. B39, 493-518.

Eynde, S. V., Mathot, V. B. F., Koch, M. H. J. \& Reynaers, H. (2000). Polymer, 41, 4889-4900.

Goderis, B., Reynaers, H. \& Koch, M. H. J. (2002). Macromolecules, 35, 5840-5853.

Goderis, B., Reynaers, H., Koch, M. H. J. \& Mathot, V. B. F. (1999). J. Polym. Sci. B Polym. Phys. 37, 1715-1738.

Hosemann, R. \& Bagchi, S. N. (1962). Direct Analysis of Diffraction by Matter. Amsterdam: North Holland.

Mandelkern, L. (1992). Chemtracts Macromol. Chem. 3, 347-375.

Mathot, V. B. F. (1986). Polymer, 27, 969-970.

Mathot, V. B. F. (1994). Calorimetry and Thermal Analysis of Polymers, edited by V. B. F. Mathot, pp. 105-167. New York: Hanser Publishers.

Orwoll, R. A. \& Flory, P. J. (1967). J. Am. Chem. Soc. 89, 6814-6822. Pedersen, J. S. (2004). J. Appl. Cryst. 37, 369-380.

Peeters, M., Goderis, B., Vonk, C., Reynaers, H. \& Mathot, V. (1997). J. Polym. Sci. B Polym. Phys. 35, 2689-2713.

Piorkowska, E. \& Galeski, A. (1993). J. Polym. Sci. B Polym. Phys. 31, 1285-1291.

Porod, G. (1951). Kolloid Z. 124, 83-114.

Roe, R. J. (2000). Methods of X-ray and Neutron Scattering in Polymer Science. New York: Oxford University Press.

Ruland, W. (1977). Colloid Polym. Sci. 255, 417-427.

Strobl, G. R. \& Schneider, M. (1980). J. Polym. Sci. Polym. Phys. Ed. 18, 1343-1359.

Swan, P. R. (1960). J. Polym. Sci. 42, 525-534.

Varga, J. \& Ehrenstein, G. W. (1996). Polymer, 37, 5959-5963.

Vonk, C. G. \& Kortleve, G. (1967). Kolloid Z. Z. Polym. 220, 19-24.

Vonk, C. G. \& Pijpers, A. P. (1985). J. Polym. Sci. Polym. Phys. 23, $2517-2537$

Vonk, C. G. \& Reynaers, H. (1990). Polym. Commun. 31, 190-193.

Zhukov, A. A., Perkins, G. K., Thomas, J. V., Caplin, A. D., Küpfer, H. \& Wolf, T. (1997). Phys. Rev. B, 56, 3481-3487. 